\title{
Youth priorities for global health
}

\author{
Young people must be involved in setting the global health agenda.
}

C OVID-19 is a pandemic that has taken the world by storm, and that has an uncertain end to it. It is therefore understandable that the global health community has diverted all its efforts into understanding the virus and its origin. However, COVID-19 research has led to a diversion of efforts from other health conditions that continue to contribute to a large disease burden, especially noncommunicable diseases (NCDs).

A population for whom addressing NCDs is of utmost importance is young people. Young people contribute to the global health discourse as researchers, practitioners, patients, activists and community organizers. However, their needs and perspectives are not prioritized.

Various NCDs, such as cardiovascular disease, diabetes, cancer and obesity, affect children and young people. Exposure to risk factors initiated in adolescence leads to the adoption of unhealthy behaviors for life, which results in the risk of developing NCDs in adulthood. For instance, NCD risk factors, such as unhealthy dietary habits and alcohol consumption, if unaddressed, risk storing up a health crisis for the next generation; many of these risk factors have been exacerbated during this pandemic. The situation is critical in low- and middle-income countries (LMICs) where the health systems are less resilient.

In 2011, the World Health Organization undertook an extensive exercise to establish a prioritized research agenda for NCDs. Various target groups were prioritized, but the research agenda neglected an important constituency: adolescents and young people.

This lack of focus on young people's health and wellbeing is reflected in the research, with only $2.2 \%$ of the total development assistance for health being spent on adolescent health. NCD research on adolescents and young people is limited, and for whatever is available, there are little disaggregated data by gender and age, or on special groups such as those in humanitarian crisis or with disabilities, which makes it difficult to plan programs and interventions. Where research does exist, it is often from high-income settings.

The lack of evidence on effective and cost-effective interventions in young people in LMICs hampers the ability to generate evidence that can result in effective implementation and policy changes.

It would be unfair to say that nothing has been done so far, but existing efforts are disjointed and lack coherence. The World Health Organization's maternal, newborn, child and adolescent health portal maps mortality, morbidity, risk factors, coverage, and policy on maternal, newborn, child and adolescent health, but the implementation, monitoring and evaluation of these policies at the national level remains untracked. The availability of standardized age-disaggregated data also remains a substantial challenge in many countries. This inability to measure improvements in young people's health disincentivizes governments from investing in the health of young people and thereby ensuring sustainable programs, interventions and policies.

Just as the health needs of young people are not reflected in data and research, their voices and insights are also frequently excluded from policy discourse and governance mechanisms. I know from my own experience as a young advocate for NCDs that getting young people included as a key aspect of the agenda is a real struggle.

Although United Nations agencies and some national governments talk a lot about meaningful inclusion, the reality is quite different. Young people are not well represented as a constituency at national and global fora, with their participation often limited to a mere tokenism, rather than being recognized as a powerful force for accountability. Senior researchers usually get priority, with young people left behind, seldom recognized for their potential.

The opportunities are even fewer for engaging young people in research and data collection, opportunities that are crucial for their personal development as early-career researchers. Young people are already working in their communities and driving change. Sharing the experiences of early-career researchers through the constituency itself is warranted and could help in the design of programs and policies for young people at the national and global levels. A collective enabling space for youth advocacy at the global level is also needed.

Efforts are being made to bring various youth actors together on a common platform and to move away from a siloed approach. To truly leverage the potential of youth-led organizations, a youth coalition on NCDs has been convened under the aegis of UN Major Group for Children and Youth, which is an official and self-organized mechanism for young people to meaningfully engage in the United Nations and holds a formal mandate in the follow-up and review of its Sustainable Development Goals. The goals of this coalition are to share expertise and experience, work strategically and collaboratively to strengthen the youth NCD movement, set priorities for action, and develop a joint research and advocacy platform to improve the health, well-being and human rights of all young people. The coalition draws strength from diverse youth constituencies, including youth practitioners, activists and patient advocates.

Young people are critical players in the global health agenda. Leaving them out of decision-making will substantially hamper progress in achieving the sustainable development goals, whereas involving them will help in realizing a healthier, more equitable world.

\section{Ishu Kataria (D)}

Center for Global Noncommunicable Diseases, RTI International, Aerocity, New Delhi, India. $凶_{e-m a i l: i k a t a r i a @ r t i . o r g}$

Published online: 13 September 2021 https://doi.org/10.1038/s41591-021-01474-8

Competing interests

The author declares no competing interests. 